\title{
CONFIABILIDAD DE ESTRUCTURAS CONSIDERANDO EL DAÑO ACUMULADO EN EL TIEMPO
}

\author{
Dante Tolentino López ${ }^{(1)}$, Sonia Elda Ruiz Gómez ${ }^{(2)}$, Marco Antonio Torres Pérez-Negrón ${ }^{(3)}$, Marco \\ Antonio Montiel Ortega ${ }^{(4)}$
}

\section{RESUMEN}

Se propone un criterio para evaluar la confiabilidad estructural en el que se utiliza una expresión matemática que considera la acumulación de daño por sismo en un intervalo de tiempo. Dicha expresión toma en cuenta de manera simultánea, la variación en el tiempo de la capacidad estructural y de la demanda sísmica para una intensidad dada. En la formulación se consideran las incertidumbres epistémicas asociadas a la capacidad y a la demanda, así como la aleatoriedad de los eventos sísmicos y de la respuesta estructural. La confiabilidad se expresa en términos del número esperado de fallas al término de un intervalo de tiempo, correspondiente a diferentes estados límite. El criterio se ejemplifica mediante el análisis de un edificio de 10 niveles ubicado en suelo blando con características similares al sitio de la Secretaría de Comunicaciones y Transportes en la Ciudad de México.

Palabras Clave: confiabilidad estructural; deterioro; demanda estructural; daño acumulado

\begin{abstract}
A criterion to evaluate the seismic structural reliability considering the accumulation of seismic damage is proposed using a closed mathematical expression. This expression takes into account the variation of the structural capacity and of the demand for a given intensity over time. The formulation considers epistemic uncertainties associated to capacity and demand, and randomness of seismic events. The structural reliability is expressed in terms of the expected number of failures at the end of a time interval, for different limit states. The criterion is exemplified by the analysis of a 10-story building located on soft soil with characteristics similar to those of the Ministry of Communications and Transports site in Mexico City.
\end{abstract}

Key Words: structural reliability; deterioration; structural demand; cumulative damage

Artículo recibido el 2 de septiembre de 2010 y aprobado para su publicación el 11 de febrero de 2011.

(1) Estudiante de Posgrado, Mecánica Aplicada, Instituto de Ingeniería, UNAM, Coyoacán,04510 México, D.F. Teléfono: (55) 5623-36-00 ext 8480; dtolentinol@iingen.unam.mx

(2) Investigadora, Mecánica Aplicada, Instituto de Ingeniería, UNAM, Coyoacán,04510 México, D.F. Teléfono: (55) 5623 36 54; sruiz@ iingen.unam.mx

(3) Coordinador de Proyectos de Ingeniería, ERN Ingenieros Consultores S.C., Vito Alessio Robles No.179 Col. Hda. de Gpe. Chimalistac. Álvaro Obregón, 01050, México, D.F. Teléfono: (55) 5616-8161, 62, 64, ext. 103; mtorres@ern.com.mx

(4) Profesor, Posgrado de Ingeniería, Universidad Autónoma de Querétaro; mmo@uaq.mx 


\section{INTRODUCCIÓN}

La variación de las propiedades dinámicas estructurales en el tiempo es un problema común al que se encuentra expuesta toda obra de Ingeniería Civil de cualquier país. Las estructuras deben mantenerse dentro de niveles de desempeño adecuados para garantizar su funcionalidad y evitar los costos humanos, económicos y sociales asociados a un mal comportamiento a lo largo de toda su vida útil. A medida que el daño en la estructura se acumula, también se incrementa la probabilidad de que incurra en un nivel de desempeño no adecuado.

Al existir una disminución de la rigidez y de la resistencia de la estructura, también existe una variación en la respuesta que la excitación sísmica le demanda a la estructura. Esto implica que entre mayor sea el daño de la estructura menor será la intensidad sísmica necesaria para sobrepasar una cierta condición de desempeño.

Lo anterior hace necesario desarrollar expresiones, herramientas y metodologías para estimar las variaciones en los niveles de confiabilidad a los que estará sometida una estructura a lo largo de su vida útil, tomando en cuenta que tanto su capacidad como su demanda (ante cierta intensidad) varían a lo largo del tiempo.

Con la finalidad de estudiar la influencia del daño acumulado en la confiabilidad estructural, varios autores han desarrollado metodologías modelando dicho daño mediante procesos de Markov (Hamammoto, 1996; Gusella, 1998; Montes-Iturrizaga et. al., 2003; Cruz, 2007), así como mediante simulaciones de Monte Carlo (Díaz y Esteva, 1997; Vásquez, 2010).

Aquí se presenta un criterio para evaluar la confiabilidad estructural en el que se toma en cuenta la variación en el tiempo tanto de la capacidad estructural como de la demanda dada una intensidad. La confiabilidad se expresa en términos del número esperado de fallas en un intervalo de tiempo, $\bar{\eta}_{F, T}$. El criterio que aquí se presenta se basa en las expresiones del método simplificado propuesto por Cornell y colaboradores (2002), pero que aquí se extiende para considerar que las propiedades estructurales cambian con el tiempo. Se hace ver que para evaluar la confiabilidad estructural no basta con considerar que la capacidad de la estructura se reduce, sino que también se debe tomar en cuenta que el deterioro estructural influye en la demanda de la estructura (que se encuentra sujeta a excitaciones de cierta intensidad). El criterio propuesto se ejemplifica mediante el análisis de la confiabilidad de un edificio de concreto reforzado de 10 niveles y tres crujías localizado en la zona IIIb del valle de México.

\section{EVALUACIÓN DE LA CONFIABILIDAD ESTRUCTURAL EN EL TIEMPO}

\section{Influencia y aleatoriedad de parámetros implícitos en la demanda y la capacidad}

La confiabilidad estructural normalmente se ve influenciada por parámetros inciertos relacionados con la capacidad y con la demanda estructural (Cornell, 1996; Wen, 1995). Debido a las limitaciones en información y a las metodologías empleadas para evaluar la demanda y la capacidad estructural, se identifican los siguientes tipos de incertidumbres (asociados tanto a la capacidad $C$ como a las demanda $D)$ :

$\underline{\text { La aleatoriedad }}$. Se refiere a la variabilidad de los fenómenos físicos (cargas vivas, oleaje, sísmicas, nieve, granizo, viento, etc.) que afectan tanto a la demanda como a la capacidad estructural. Estas incertidumbres se pueden referir a magnitud, duración, tiempo de ocurrencia, etc. y pueden 
cuantificarse con menor o mayor detalle dependiendo de la información disponible y del grado de exactitud de los modelos matemáticos utilizados.

Las incertidumbres epistémicas. Son aquellas incertidumbres que resultan de la falta de conocimiento o información que se presenta al cuantificar las magnitudes y distribuciones de cargas, así como las consideraciones y restricciones que se toman al modelar la estructura.

\section{Metodología para obtener el número de fallas en un intervalo de tiempo}

Para calcular la tasa media anual de falla de una estructura con propiedades determinísticas en sus propiedades mecánicas (asociada a cierto estado límite), se puede utilizar la siguiente ecuación (Cornell, 1968; Esteva, 1968):

$v_{F}(c)=\int_{0}^{\infty}\left|\frac{d v(y)}{d y}\right| P[c<S \mid y] d y$

donde $\left|\frac{d v(y)}{d y}\right|$ representa el valor absoluto de la derivada de la curva de peligro sísmico (es necesario considerar el valor absoluto, debido a que la derivada es negativa), $P[c<S \mid y]$ es la probabilidad de que la capacidad de la estructura asociada al estado limite c, sea menor que la solicitación a la que se somete el sistema, dada una intensidad, $y$.

En el caso que se tomen en cuenta las incertidumbres relacionadas con la capacidad de la estructura, el valor esperado de $v_{F}$ se expresa de la siguiente forma:

$E\left[v_{F}\right]=\int_{0}^{\infty} \int_{0}^{\infty}\left|\frac{d v(y)}{d y}\right| P[C<S \mid y, c] f_{C}(c) d y d c$

donde $P[C<S \mid y, c]$ es la probabilidad de falla cuando ocurre un evento de intensidad $y$, y $f_{C}(c)$ es la función de densidad de probabilidades de la capacidad estructural del estado límite en estudio.

Si además se desea tomar en cuenta la variación de la capacidad estructural en el tiempo, $\tau$, se deberá considerar que la capacidad del estado límite de estudio, $C(\tau)$, así como su función de densidad de probabilidades, varían con el tiempo. Por lo tanto, el valor esperado del número de fallas para el intervalo de tiempo $[0, \Delta t)$ es:

$E\left[\eta_{F, T}(0, \Delta t)\right]=\int_{0}^{\Delta t} \int_{0}^{\infty} \int_{0}^{\infty}\left|\frac{d v(y)}{d y}\right| P[C(\tau)<S \mid y, \tau] f_{C}(c \mid \tau) d y d c d \tau$

donde $P[C(\tau)<S \mid y, \tau]$ es la probabilidad de falla cuando ocurre un evento de intensidad $y$, y $f_{C}(c \mid \tau)$ es la función de densidad de probabilidades de la capacidad estructural del estado límite en un instante de tiempo $\tau$. Si $\tau=0$, la estructura no presenta ningún cambio en sus propiedades; sin embargo, a medida que transcurre el tiempo $\tau$, la estructura va acumulando daño y, como consecuencia, se reduce su capacidad estructural para el estado límite en estudio. 
El número esperado de fallas durante un intervalo de tiempo es igual a la tasa de falla integrada para ese intervalo de tiempo. Entonces, si se considera la variación de la capacidad en un intervalo de tiempo $[t, t+\Delta t)$, el valor esperado de número de fallas se expresa como:

$E\left[\eta_{F, T}(t, \Delta t)\right]=\int_{t}^{t+\Delta t} \int_{0}^{\infty} \int_{0}^{\infty}\left|\frac{d v(y)}{d y}\right| P[C(\tau)<S \mid y, \tau] f_{C}(c \mid \tau) d y d c d \tau$

Si además se considera que la demanda estructural dada una intensidad es aleatoria con cierta función de probabilidad en un instante de tiempo $\tau$ igual a $f_{S}(s \mid y, \tau)$, entonces:

$$
E\left[\eta_{F, T}(t, \Delta t)\right]=\int_{t}^{t+\Delta t} \int_{0}^{\infty} \int_{0}^{\infty} \int_{0}^{\infty}\left|\frac{d v(y)}{d y}\right| P[C(\tau)<S(\tau) \mid y, \tau] f_{C}(c \mid \tau) f_{S}(s \mid y, \tau) d y d c d s d \tau
$$

\section{Método simplificado}

Con el fin de no realizar las integrales que se presentan en las ecuaciones 4 y 5 enseguida se presenta el método simplificado propuesto por Cornell y colaboradores (2002) que aquí se extiende para tomar en cuenta el efecto del deterioro de las propiedades estructurales en la evaluación de la confiabilidad.

Las integrales obtenidas en la ec. 4 se pueden simplificar con respecto a las variables y y $c$ si se hacen las siguientes consideraciones:

- La curva de peligro sísmico $(C P S), v_{Y}(y)$, se puede representar para la intensidad de interés, mediante la expresión $v_{Y}(y)=k \cdot y^{-r}$, donde $r$ y $k$ son parámetros que se ajustan a la forma de la curva de peligro sísmico.

- La capacidad estructural del estado límite en el tiempo $\tau$, presenta una mediana de capacidad, $\hat{C}(t)$, de tipo lognormal y desviación estándar del logaritmo natural es igual a $\sigma_{\operatorname{lnC} \mid \tau}$.

- La mediana de la demanda estructural $\mathcal{D}$, se representa como función de la intensidad, $y$, mediante la expresión $\hat{D}=a \cdot y^{b}$ donde $a$ y $b$ son parámetros que se ajustan a la $C P S$. Se considera que la demanda estructural para cierta intensidad $y$ tiene una distribución lognormal con desviación estándar del logaritmo natural igual a $\sigma_{l n D \mid y_{\hat{C}, \tau}}$. (Rosenblueth y Esteva, 1972; Shome y Cornell, 1999).

Considerando lo anterior, se obtiene la siguiente expresión para el valor esperado del número de fallas durante el intervalo $[t, t+\Delta t$ ) (Torres y Ruiz, 2007):

$\bar{\eta}_{F, T}(t, \Delta t)=\int_{t}^{t+\Delta t} k\left(y_{\hat{C, \tau}}\right)^{-r} \exp \left[\frac{r^{2}}{2 b(\tau)^{2}}\left(\sigma_{\ln D \mid y_{\hat{C}, \tau}}^{2}+\sigma_{\ln C \mid \tau}^{2}+\sigma_{U D \mid \tau}^{2}+\sigma_{U C \mid \tau}^{2}\right)\right] d \tau$

donde $y_{\hat{C}, \tau}=\left[\frac{\hat{C}(\tau)}{a(\tau)}\right]^{\frac{1}{b(\tau)}}$ es la intensidad de la carga sísmica asociada con la mediana de la capacidad, $\hat{C}(\tau)$, en el tiempo $\tau ; \sigma_{\ln D \mid y_{\hat{C}, \tau}^{2}} y \sigma_{\ln C \mid \tau}^{2}$ son las desviaciones estándar de los logaritmos naturales de la demanda, $D$, y de la capacidad estructural, $C$, del estado límite de interés, en el tiempo $\tau ; \sigma_{U D \mid \tau}^{2} y \sigma_{U C \mid \tau}^{2}$ son las 
incertidumbres epistémicas relacionadas con la demanda estructural y la capacidad estructural del estado límite de interés, en el tiempo $\tau$, respectivamente.

Para resolver la integral de la ec. 6 se hacen las siguientes simplificaciones:

- La mediana de la capacidad, $\hat{C}$, varía en el tiempo de la forma:

$$
\hat{\mathrm{C}}(\tau)=\alpha+\beta(\tau)
$$

donde $\alpha>0$ y $\beta<0$

- Los valores de $\sigma_{\ln C \mid \tau}$ y $\sigma_{\left.\ln D\right|_{\hat{C}, \tau}}$ se consideran constantes en el intervalo de tiempo, $\Delta t$, expresándose de la siguiente forma:

$$
\sigma_{\ln D \mid y_{\hat{C}, \tau}}=\sigma_{\ln D \mid y_{\hat{C}, t}}
$$

$\sigma_{\operatorname{lnC} \mid \tau}=\sigma_{\operatorname{lnC} \mid t}$

- Los parámetros $a(\tau)$ y $b(\tau)$ tienen valores constantes durante el intervalo de tiempo $\Delta t$, por lo que:

$a(\tau)=a_{t}$

$b(\tau)=b_{t}$

Anteriormente, Torres (2006), y Torres y Ruiz (2007) encontraron que el valor esperado del número de fallas considerando la variación de la capacidad para un intervalo de tiempo se expresa como:

$\bar{\eta}_{F, T}(t, \Delta t)=k\left(y_{\hat{C} \mid \tau}\right)^{-r} \exp \left[\frac{r^{2}}{2 b_{t}^{2}}\left(\sigma_{\ln D \mid y_{\hat{C}, t}^{2}}^{2}+\sigma_{\ln C \mid t}^{2}+\sigma_{U D \mid t}^{2}+\sigma_{U C \mid t}^{2}\right)\right] \Omega(t, \Delta t)$

para la cual:

$$
y_{\hat{C} \mid t}=\left[\frac{\hat{C}(t)}{a_{t}}\right]^{\frac{1}{b_{t}}}
$$

$\Omega(t, \Delta t)=\frac{(\alpha+\beta t)}{\beta} \frac{b_{t}}{\left(b_{t}-r\right)}\left[\left(1+\frac{\beta \Delta t}{\alpha+\beta t}\right)^{1-\frac{r}{b_{t}}}-1\right]$

donde $\Omega(t, \Delta t)$ es el factor de corrección del número esperado de fallas cuando se considera la variación de la capacidad estructural.

En el presente estudio se extiende la formulación anterior para hacer intervenir, además, la alteración que sufre la demanda estructural debido a la degradación de las propiedades mecánicas de la estructura en el tiempo. Para ello, con el fin de resolver la ec. 6 es necesario hacer las siguientes consideraciones: 
- Los valores de la mediana de la demanda en el tiempo se obtienen mediante la siguiente expresión

$$
\hat{D}=a(\tau) \cdot y^{b}+g \cdot \tau
$$

donde $a(\tau)=e+f \cdot \tau$, quedando la expresión igual a:

$$
\hat{D}=(e+f \cdot \tau) \cdot y^{b}+g \cdot \tau
$$

donde $e, f, b$, y $g>0$

Nótese que en la formulación anterior la ecuación 15 estaba dada por $\hat{D}=a \cdot y^{b}$.

La forma de la ecuación 16 se propuso con base en los resultados obtenidos del ejemplo que se presenta en la siguiente sección. Los parámetros $e+f \cdot \tau$ representan la forma en la que se van incrementando los valores de la demanda estructural a medida que crece el intervalo de tiempo (a medida que se va acumulando más daño en la estructura), el parámetro $g \cdot \tau$ describe el daño "inicial" que presenta la estructura (al final de cierto intervalo de tiempo e inicio del siguiente). La forma de la ecuación 16 tiene la ventaja de que conduce a una solución cerrada de la integral expresada por la ecuación 5.

Sabiendo que $y_{\hat{C}, \tau}=\left[\frac{\hat{C}(\tau)}{a(\tau)}\right]^{\frac{1}{b(\tau)}}$ es un nivel de intensidad $y$ que uno anticipa que causará una demanda (por ejemplo de distorsión máxima de entrepiso igual a la mediana de la capacidad $\mathcal{C}$, entonces $\hat{C}=\hat{D}$ (Cornell, 1999), por lo que sustituyendo $\hat{C}$ en la ec. 15, resulta:

$\hat{\mathrm{C}}(\tau)=a(\tau) \cdot y^{b}+g \cdot \tau$

Despejando $y$ de la ecuación anterior, queda la siguiente expresión:

$y=\left(\frac{\hat{C}(\tau)-g \cdot \tau}{a(\tau)}\right)^{\frac{1}{b}}$

Sustituyendo el valor de $\hat{C}$ (ec. 7) en la ec. 18:

$y=\left(\frac{\alpha+\beta \cdot \tau-g \cdot \tau}{\alpha(\tau)}\right)^{\frac{1}{b}}$

Dado que $\beta$ y $g$ son constantes, entonces:

$y=\left(\frac{\alpha+\tau(\beta-g)}{a(\tau)}\right)^{\frac{1}{b}}$

Haciendo $\beta^{\prime}=(\beta-g)$, finalmente se obtiene la siguiente expresión de $y_{\hat{C}, D \mid \tau}$ : 
$y_{\hat{C}, D \mid \tau}=\left(\frac{\alpha+\beta^{\prime} \cdot \tau}{e+f \cdot \tau}\right)^{\frac{1}{b}}$

De aquí se tiene que el valor esperado del número de fallas considerando la variación de la capacidad y de la demanda estructural dada una intensidad, para un intervalo de tiempo, resulta como sigue:

$\bar{\eta}_{F, T}(t, \Delta t)=k\left(y_{\hat{C}, D \mid t}\right)^{-r} \exp \left[\frac{r^{2}}{2 b(t)^{2}}\left(\sigma_{l n D \mid y_{\hat{C}, t}}^{2}+\sigma_{l n C \mid t}^{2}+\sigma_{U D \mid t}^{2}+\sigma_{U C \mid t}^{2}\right)\right] \Omega_{0}(t, \Delta t)$

donde:

$\Omega_{0}(t, \Delta t)=$

$-\frac{b\left(\text { Hypergeometric } 2 \mathrm{~F} 1\left[1-\frac{r}{b^{\prime}},-\frac{r}{b^{\prime}}, 2-\frac{r}{b^{\prime}} \frac{f\left(\alpha+\beta^{\prime} t\right)}{f \alpha-e \beta^{\prime}}\right]\right)\left(\frac{(e+f t) \beta^{\prime}}{-f \alpha+e \beta^{\prime}}\right)^{-\frac{r}{b}}(\alpha+t \beta)}{(b-r) \beta}+$

$\frac{b\left(\frac{\alpha+t \beta^{\prime}}{e+f t}\right)^{\frac{r}{b}}\left(\text { Hypergeometric2F1 }\left[1-\frac{r}{b^{\prime}}, \frac{r}{b^{\prime}}, 2-\frac{r}{b^{\prime}}, \frac{f\left(\alpha+\beta^{\prime}(t+\Delta t)\right)}{f \alpha-e \beta^{\prime}}\right]\right)\left(\frac{\beta^{\prime}(e+f(t+\Delta t))}{-f \alpha+e \beta^{\prime}}\right)^{-\frac{r}{b}}\left(\alpha+\beta^{\prime}(t+\Delta \mathrm{t})\right)\left(\frac{\alpha+\beta^{\prime}(t+\Delta t)}{e+f(t+\Delta t)}\right)^{-\frac{r}{b}}}{(b-r) \beta^{\prime}}$

donde $\Omega_{0}(t, \Delta t)$ es el factor de corrección del número esperado de fallas al final de un intervalo de tiempo cuando se considera la variación en el tiempo de la capacidad y de la demanda estructural sísmica para una intensidad dada.

\section{EJEMPLO DE APLICACIÓN}

\section{Descripción de la estructura}

La expresión propuesta (ec. 22) se aplica a un edificio de concreto reforzado de 10 niveles y tres crujías. Para el diseño del edificio se empleó el Reglamento de Construcciones del Distrito Federal (RCDF 2004) y sus Normas Técnicas Complementarias para Diseño por Sismo (NTCS, 2004). El edificio fue diseñado por García Jarque Ingenieros, S.C.

El edificio es una estructura regular a base de marcos de concreto reforzado, con una planta cuadrada de $30 \mathrm{~m}$ formada por tres crujías de $10 \mathrm{~m}$, y con una altura de entrepiso de $4 \mathrm{~m}$. (ver figura 1). Se considera ubicado en un sitio con condiciones similares al de la Secretaría de Comunicaciones y Transportes (SCT, Zona IIIb). Se diseñó con un factor de comportamiento sísmico $Q=3$ y se utilizó un coeficiente sísmico de diseño $C s=0.45$. 

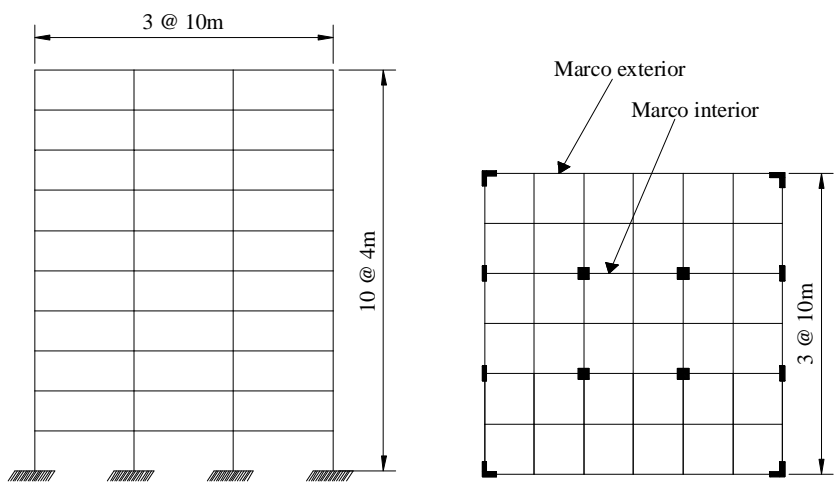

Figura 1. Elevación y planta del edificio estudiado.

Para efectuar el análisis no-lineal se modeló el edificio mediante un marco interior y uno exterior. Estos se consideraron unidos con barras articuladas en los extremos que solo tienen rigidez axial (ver figura 2). El sistema estructural (cuando se consideran sus propiedades medias) tiene un periodo fundamental de vibración de T=1.1s (Montiel, 2006).

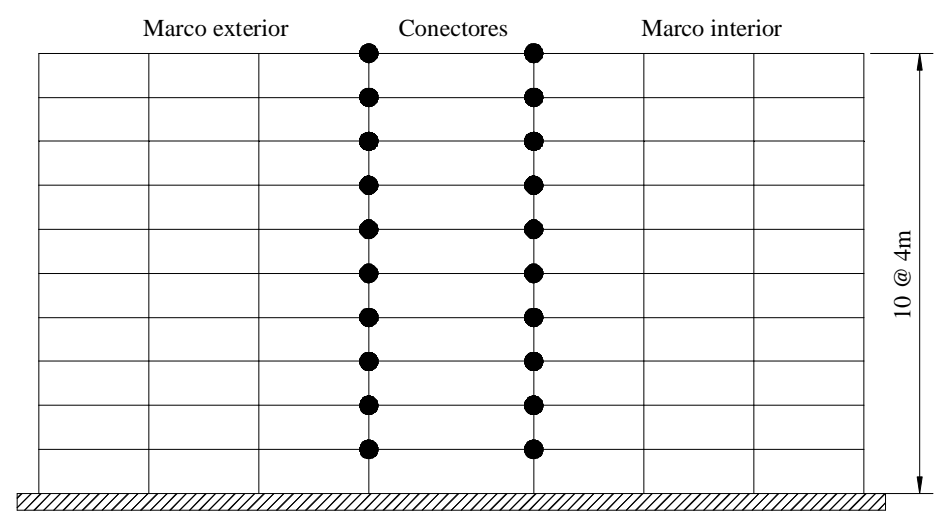

Figura 2. Modelo estructural.

En la tabla 1 se muestran los valores nominales de las secciones de trabes y columnas. Se usó una resistencia a la compresión del concreto f $f^{\prime} c=250 \mathrm{~kg} / \mathrm{cm}^{2}$ y esfuerzo de fluencia del acero fy=4200 kg/cm 2 .

Tabla 1. Secciones nominales del edificio en estudio.

\begin{tabular}{ccccc}
\hline Marco & Nivel & Trabe $(\mathrm{cm})$ & Columna interior $(\mathrm{cm})$ & Columna exterior $(\mathrm{cm})$ \\
\hline Interior & $\begin{array}{c}1-7 \\
8-10\end{array}$ & $35 \times 90$ & $\begin{array}{c}110 \times 110 \\
90 \times 90\end{array}$ & $150 \times 50$ \\
\hline Exterior & $1-10$ & $45 \times 130$ & $50 \times 150$ & $150 \times 150 \times 50$ (Sección L) \\
\hline
\end{tabular}

\section{MOVIMIENTOS SÍSMICOS}

Con el fin de realizar el análisis probabilístico de la estructura es necesario contar con un número suficiente de movimientos sísmicos intensos en el sitio en donde se encuentra la estructura (por lo que más 
adelante se simula un conjunto de acelerogramas sintéticos). Por otro lado, también es necesario simular los tiempos de ocurrencia y las intensidades de dichos sismos en el tiempo (más adelante se explican las hipótesis para realizar dicha simulación).

\section{Simulación de acelerogramas}

Debido a que se cuenta con un número limitado de registros intensos en campo, es necesario generar movimientos mediante modelos numéricos estocásticos. En este estudio se simularon 100 sismos sintéticos a partir de los registros obtenidos en la estación SCT. El procedimiento de generación de movimientos sintéticos hace la hipótesis que el movimiento sísmico que se simula sigue un proceso estocástico ergódico gaussiano no-estacionario. Los sismos se simularon empleando el procedimiento descrito en (Rivera, 2001).

\section{Simulación de tiempos de espera entre eventos sísmicos}

Para simular los tiempos de espera entre eventos se supuso que dichos tiempos presentaban una distribución de probabilidades de tipo exponencial. La simulación se realizó haciendo las siguientes consideraciones:

- El número promedio de sucesos por unidad de tiempo $\lambda$ es igual a:

$\lambda=\frac{1}{m_{T}}$

- En este estudio se considera un promedio de tiempos de espera de eventos (con magnitudes intermedias) igual a $m_{T}=5$ años. Este valor se estimó con base en el trabajo de (Hong y Rosenblueth, 1988) en donde se indica que el promedio de sismos característicos (M>7.5) es de 8 años.

\section{Simulación de intensidades}

La simulación de la intensidad de cada movimiento sísmico se realizó tomando como base la curva de peligro sísmico correspondiente al sitio y al periodo de vibración de la estructura en estudio. El procedimiento que se siguió es el siguiente:

- Se simulan números aleatorios con distribución uniforme entre 0 y 1 .

- Se calcula la tasa anual de excedencia $v$, haciendo la hipótesis que la ocurrencia de eventos es un proceso de Poisson,

- Con el valor de $v$ se entra a la curva de peligro sísmico, y se determina el valor de la intensidad.

\section{Ocurrencia de eventos sísmicos en un intervalo de tiempo}

Para evaluar la acumulación de daño en la estructura (representada en la figura 2), esta se sometió a movimientos sísmicos consecutivos que ocurren en un intervalo de tiempo dado (aquí se usaron 50 y 150 años).

En este estudio solo se incluyeron intensidades mayores que $236 \mathrm{~cm} / \mathrm{seg}^{2}$ ya que se observó que intensidades menores solo producían niveles de daño despreciables. Este valor se seleccionó a partir de la ordenada espectral para el periodo $\mathrm{T}=1.1 \mathrm{~s}$ del espectro de pseudoaceleraciones del registro obtenido en el 
sitio SCT el 19 de septiembre de 1985 en la Ciudad de México. El periodo T=1.1s corresponde al periodo fundamental del edificio en estudio. En la figura 3 se muestra un ejemplo de las intensidades de eventos simulados.

Se simularon 20 conjuntos de intensidades sísmica, de las cuales solo 8 y 15 conjuntos presentaron valores de intensidades simuladas mayores que $236 \mathrm{~cm} / \mathrm{s}^{2}$ para los intervalos de tiempo 50 y 150 años, respectivamente.

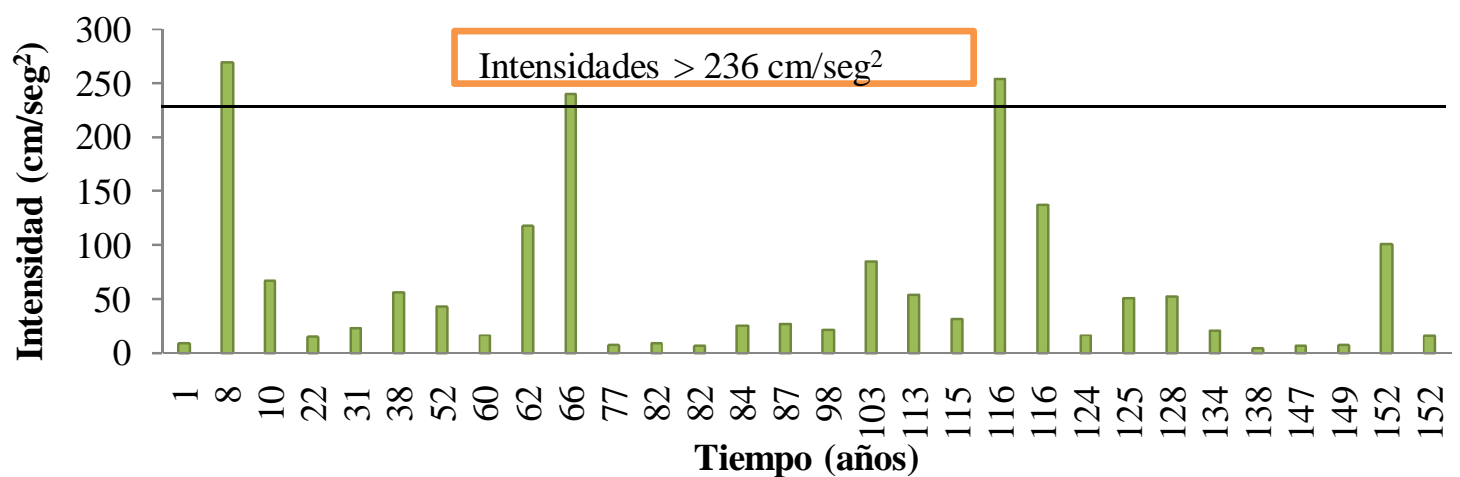

Figura 3. Ejemplos de historias de eventos simulados.

\section{EVALUACIÓN DE LA CAPACIDAD EN EL TIEMPO}

Con el fin de evaluar la capacidad de la estructura esta se excitó en la base con una secuencia de movimientos sísmicos simulados y, después de estos, se le aplicó una aceleración monotónicamente creciente simulando la acción de un análisis estático no lineal (Torres et al. 2006) (ver figura 4). Cada uno de los acelerogramas simulados se escaló de acuerdo a las intensidades simuladas previamente. En algunos conjuntos de intensidades simuladas los valores fueron menores que $236 \mathrm{~cm} / \mathrm{s}^{2}$, por lo que en estos casos únicamente se aplicó la aceleración monotónica creciente a la estructura ya que se consideró que dichos eventos no producían daño estructural.

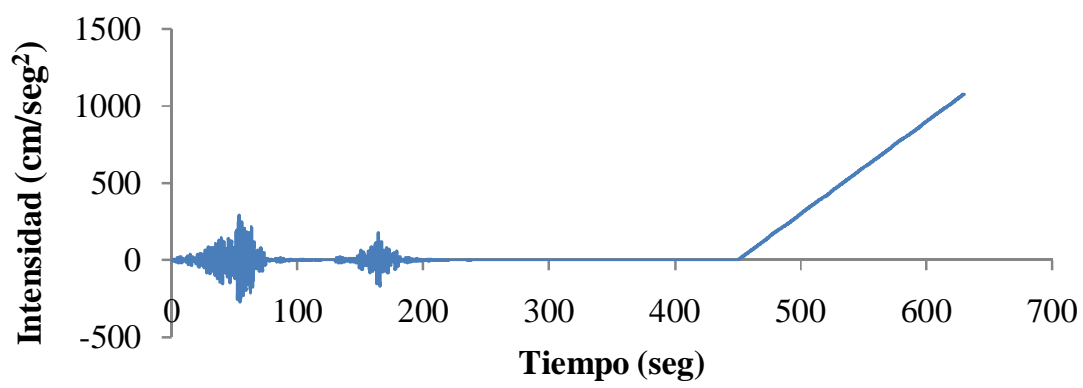

Figura 4. Ejemplo de una secuencia de dos movimientos sísmicos y una aceleración monotónica creciente al final

En las figuras $5 \mathrm{a}$ y $5 \mathrm{~b}$ se muestran las diferentes curvas de capacidad global (CC) correspondientes a distintos grupos de sismos $(1,2,6, \ldots$, etc), en un instante de tiempo de 50 años y 150 años después de construida la estructura, respectivamente. Para efectuar los análisis dinámicos no lineales se utilizó en programa Drain 2D modificado (Campos y Esteva, 1997), donde los autores incorporan al programa un 
modelo de comportamiento histerético asociado al daño que presentan los elementos de concreto reforzado. El programa Drain 2D modificado está basado en el modelo de (Wang y Shah, 1987) y modificado con base en información experimental (Townsend y Hanson, 1997; Scribner y Wight, 1978; Uzumeri, 1997; Wang y Shah, 1987; Ma et al., 1976).

En las Figuras 5 a y 5 b se puede observar la disminución que sufre la resistencia y la rigidez debido al daño originado por los sismos.

Se consideró que la falla estructural ocurría en el instante que se presentaba el mecanismo de falla, es decir, en el instante cuando se articulaban todas las vigas y columnas en un entrepiso. En la figura 6 se muestra un ejemplo de cómo se determinó la falla de la estructura para la curva de capacidad CC18, al término de 150 años después de construida la estructura (ver figura 5b).

En la tabla 2 se muestran las ductilidades disponibles globales $\mu$ obtenidas de cada curva de capacidad $C C$ \# correspondientes a 0 (sin daño), 50 y 150 años, respectivamente (figuras 5a y 5b). Las ductilidades están calculadas a partir del cociente de la distorsión máxima en la azotea entre la distorsión de fluencia del sistema.
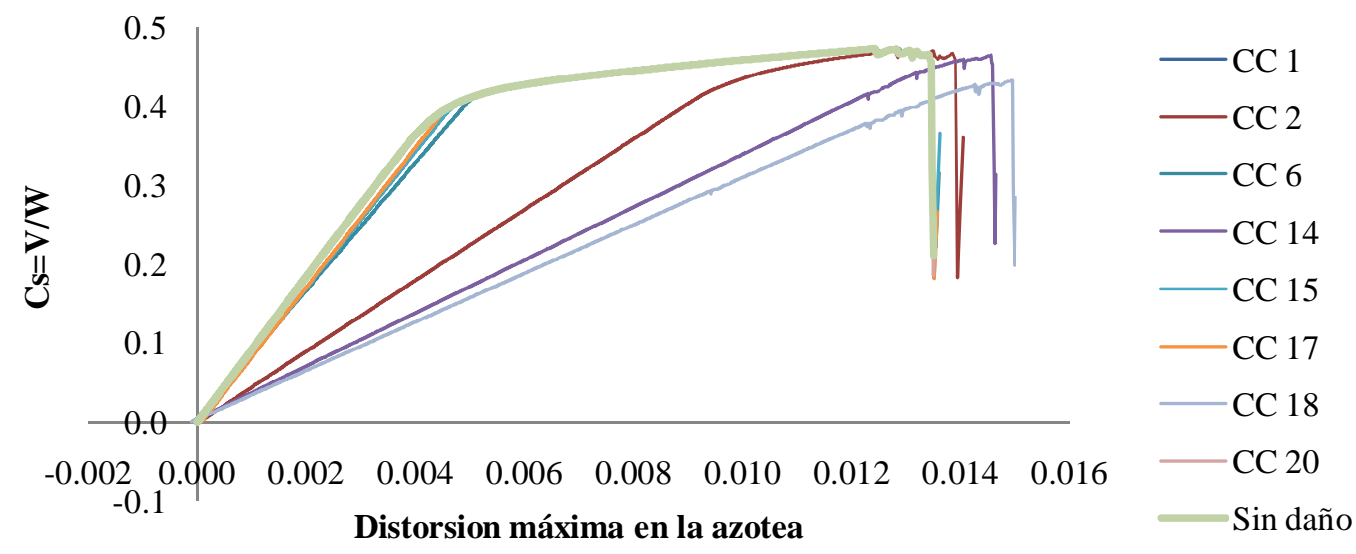

(a) 50 años

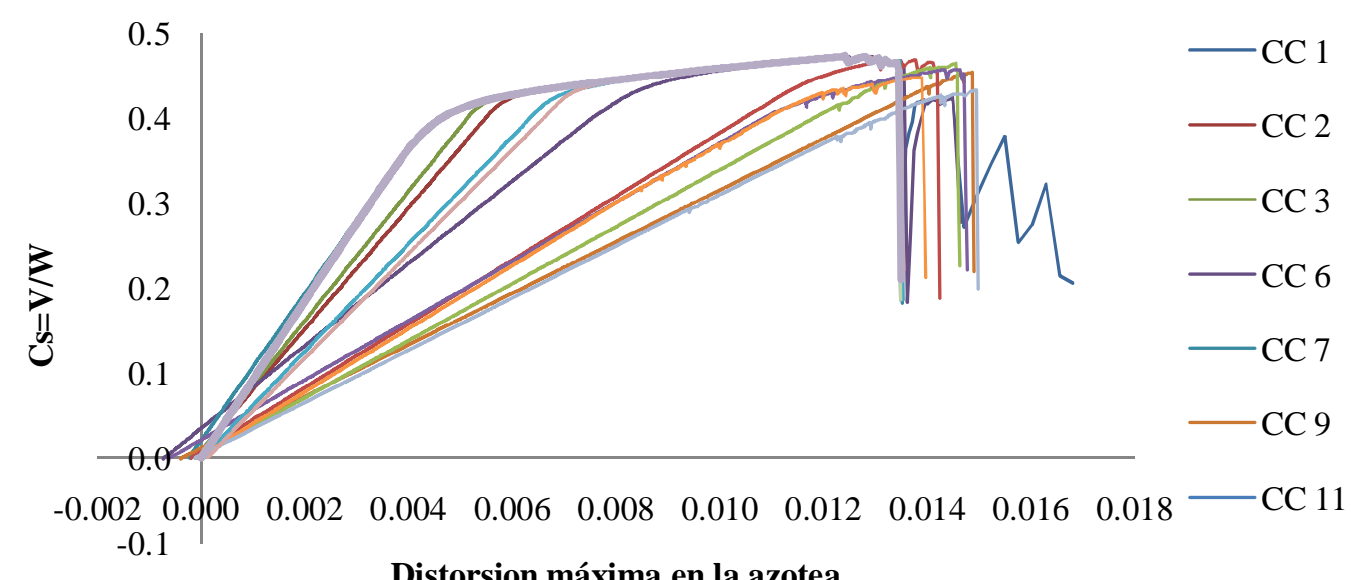

(b) 150 años

Figura 5. Curvas de capacidad correspondientes a diferentes conjuntos de eventos simulados. 


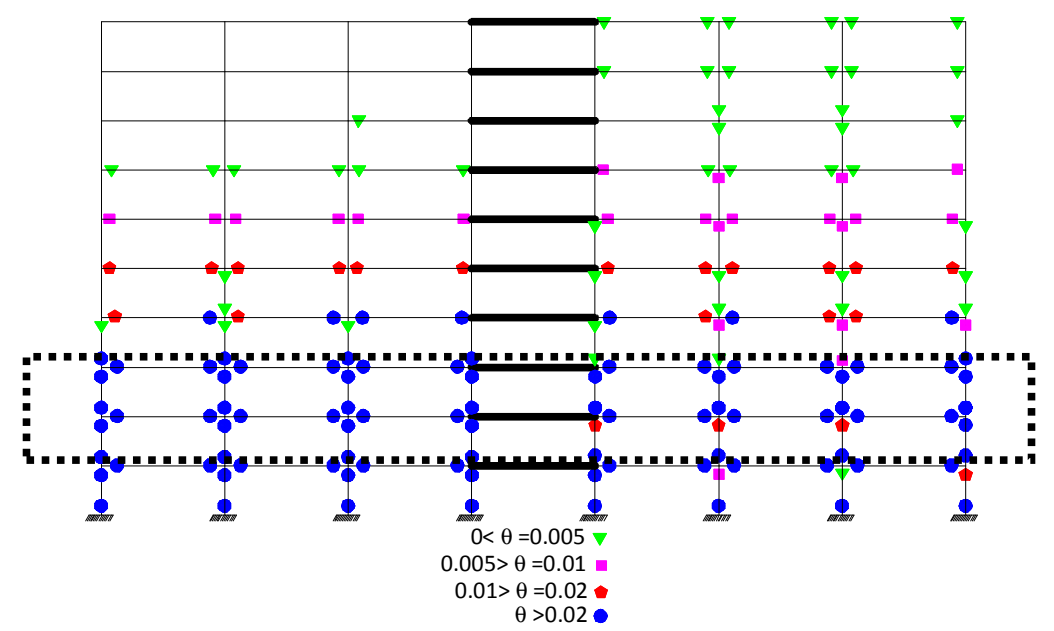

Figura 6. Mecanismo de falla (tercer entrepiso) correspondiente a la curva de capacidad CC18, asociada a un intervalo de 150 años después de construida la estructura.

Tabla 2. Ductilidades disponibles $\mu$ obtenidas a partir de las curvas de capacidad.

\begin{tabular}{cccccccccc}
\hline 0 años & \multicolumn{1}{c}{50 años } & \multicolumn{5}{c}{150 años } \\
\hline $\begin{array}{c}\text { Curva de } \\
\text { capacidad }\end{array}$ & $\mu$ & $\begin{array}{c}\text { Curva de } \\
\text { capacidad }\end{array}$ & $\mu$ & $\begin{array}{c}\text { Curva de } \\
\text { capacidad }\end{array}$ & $\mu$ & $\begin{array}{c}\text { Curva de } \\
\text { capacidad }\end{array}$ & $\mu$ & $\begin{array}{c}\text { Curva de } \\
\text { capacidad }\end{array}$ & $\mu$ \\
\hline Sin daño & 2.8 & CC 1 & 2.6 & CC 11 & 2.8 & CC 1 & 2.6 & CC 11 & 2.6 \\
& & CC 2 & 1.6 & CC 12 & 2.8 & CC 2 & 2.3 & CC 12 & 1.3 \\
& & CC 3 & 2.8 & CC 13 & 2.8 & CC 3 & 2.8 & CC 13 & 2.8 \\
& CC 4 & 2.8 & CC 14 & 1.1 & CC 4 & 2.8 & CC 14 & 1.2 \\
& CC 5 & 2.8 & CC 15 & 2.8 & CC 5 & 2.8 & CC 15 & 1.4 \\
& CC 6 & 2.8 & CC 16 & 2.8 & CC 6 & 1.8 & CC 16 & 1.9 \\
& CC 7 & 2.8 & CC 17 & 2.8 & CC 7 & 2.8 & CC 17 & 1.3 \\
& CC 8 & 2.8 & CC 18 & 1.3 & CC 8 & 2.8 & CC 18 & 1.2 \\
& CC 9 & 2.8 & CC 19 & 2.8 & CC 9 & 1.2 & CC 19 & 2.0 \\
& CC 10 & 2.8 & CC 20 & 2.6 & CC 10 & 2.8 & CC 20 & 2.6 \\
\hline
\end{tabular}

Se verificó que las ductilidades globales presentan una distribución de probabilidades de tipo lognormal. En tabla 3 se presentan los valores de la mediana de la capacidad $\hat{C}$ y de la desviación estándar del logaritmo natural para los instantes de tiempo de 0,50 y 150 años después de construida la estructura, respectivamente.

Tabla 3 Medianas de la capacidad $\hat{C}$ y desviaciones estándar del logaritmo natural $\sigma_{l n C}$ de las ductilidades disponibles globales.

\begin{tabular}{ccc}
\hline Intervalo de tiempo (años) & $\hat{C}$ & $\sigma_{l n C}$ \\
\hline 0 & 2.8 & 0 \\
50 & 2.5 & 0.2900 \\
150 & 2.0 & 0.3633 \\
\hline
\end{tabular}




\section{Obtención de los parámetros $\alpha$ y $\beta$}

El daño acumulado en un intervalo de tiempo disminuye la capacidad de la estructura (generalmente mientras más grande es el intervalo de tiempo, hay mayor disminución de la capacidad estructural). Esto es lo que se representa en la figura 7, la cual muestra la disminución de la mediana de la capacidad representada por la ductilidad disponible global , en instantes de tiempo de 0,50 y 150 años después de haber construido la estructura. También se presentan los valores de los parámetros $\alpha$ y $\beta$, ajustados de acuerdo con la ec. 7. El intervalo de tiempo igual a cero $(\tau=0)$ indica el instante que se construyó la estructura. Los otros dos intervalos indican que han pasado 50 o 150 años después de construido el edificio, y que no ha tenido ninguna rehabilitación en dichos intervalos de tiempo.

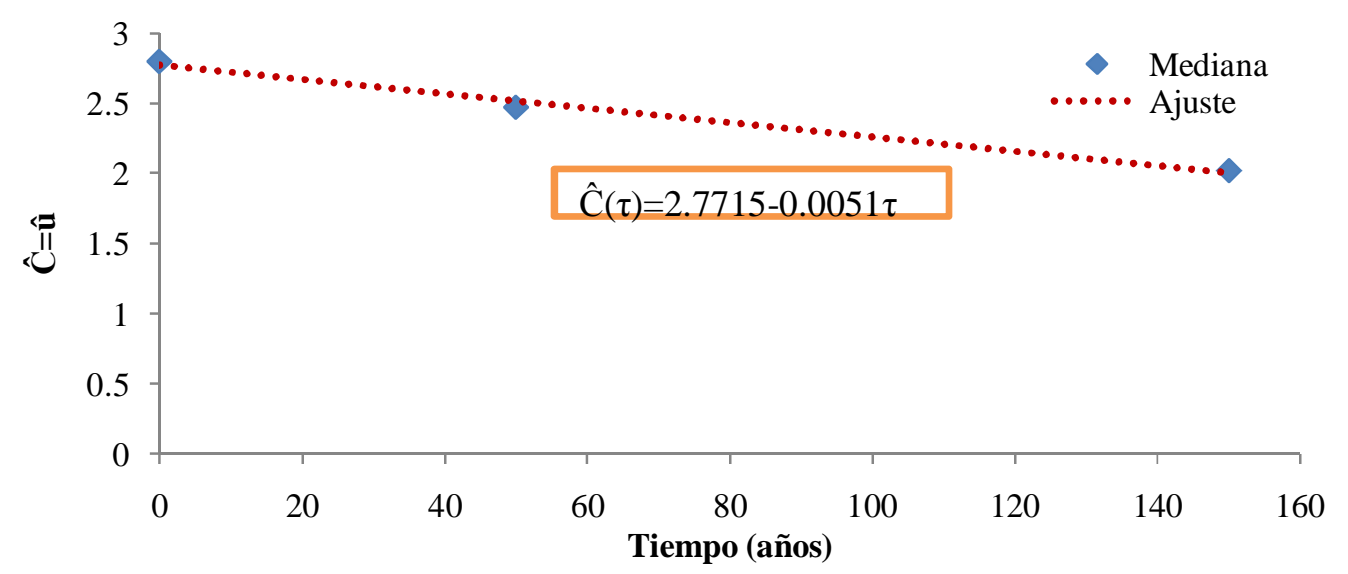

Figura 7. Mediana de la capacidad estructural, expresada en términos de la ductilidad disponible global, al término de los intervalos de tiempo de 0, 50 y 150 años.

\section{EVALUACIÓN DE LA DEMANDA ESTRUCTURAL EN EL TIEMPO}

Para evaluar la demanda estructural dada una intensidad en un instante de tiempo, la estructura se somete a una secuencia de movimientos sísmicos simulados y escalados de acuerdo con las intensidades simuladas (ver figura 8). Estos movimientos son los mismos que se usaron para determinar la capacidad estructural en los instantes de tiempo de 50 y 150 años después de haber construido la estructura. Al final de la secuencia de historias de sismos se realiza un Análisis Dinámico Incremental (Vamvatsikos y Cornell 2002). Para ello se agrega un movimiento sísmico que se escala en cada análisis hasta que la estructura llega a la falla. Para el análisis se utilizó el programa Drain 2D modificado (Campos y Esteva, 1997).

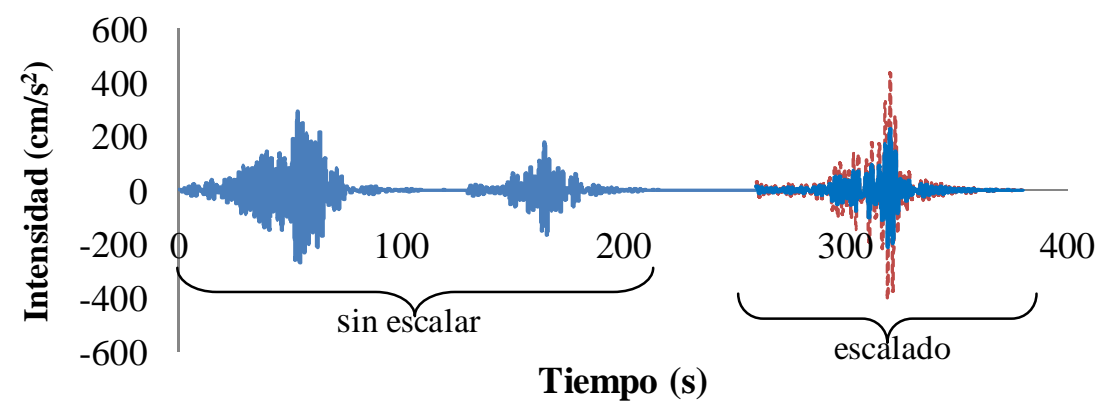

Figura 8. Serie de movimientos sísmicos para calcular la demanda estructural. 
En la figura 9 se muestran los valores de la mediana de la demanda $\hat{D}$ dada una intensidad (en función de la ductilidad global), para instantes de tiempo de 0, 50 y 150 años después de construida la estructura. A partir de los resultados se verificó que la mediana de la demanda presenta una distribución de probabilidades de tipo lognormal, para las distintas intensidades. La dispersión de los resultados se debe a la respuesta que presentan las estructuras sujetas al conjunto de historias aleatorias de aceleración.

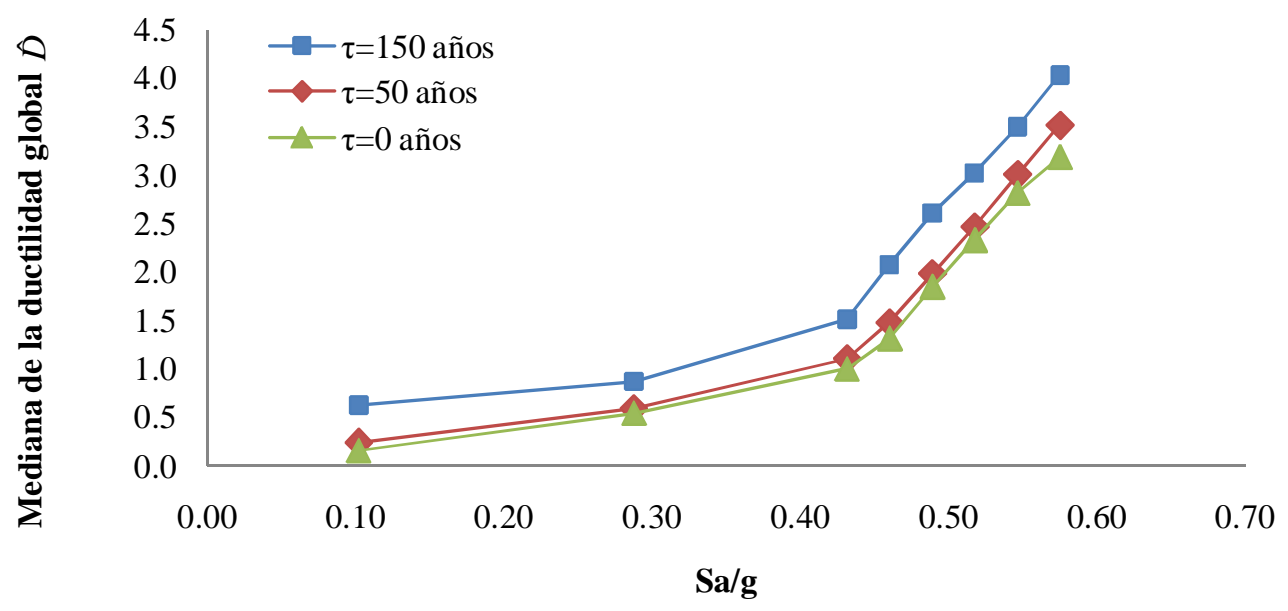

Figura 9. Valores de la mediana de la demanda $\hat{D}$, en función de la ductilidades globales, al término de los intervalos de tiempo de 0,50 y 150 años, respectivamente.

La figura 9 muestra que los valores de $\hat{D}$ para 0 años (estructura sin daño acumulado) presenta menor demanda de ductilidad. El caso contrario sucede para los instantes de tiempo de 50 y 150 años después de construida la estructura. Nótese que el daño acumulado incrementa los valores iníciales de $\hat{D}$.

El comportamiento ascendente de la curva para $\tau=0$ años (estructura sin daño acumulado) en la Figura 9, obedece al hecho que la intensidad (Sa/g) crece hacia el lado derecho del eje de las abscisas. Un comportamiento similar lo muestran las curvas correspondientes a $\tau=50$ años y $\tau=150$ años, excepto que en estos casos la ordenada al origen también se incrementa al crecer el intervalo de tiempo de interés. Dicho incremento se debe a que la estructura presenta una deformación permanente al final del intervalo de tiempo (ya sea 50 ó 150 años). Dicha deformación permanente es consecuencia del daño que ha sufrido la estructura (que presenta un comportamiento no-lineal) como consecuencia de la acción de los movimientos sísmicos intensos.

\section{Obtención de los parámetros b, e, fy $g$}

En las figuras $10 \mathrm{a}, 10 \mathrm{~b}$ y $10 \mathrm{c}$ se muestra el ajuste de la ec. 16 para al término de los intervalos de tiempo de 0,50 y 150 años, respectivamente. Los parámetros $b$, e fy g están relacionado con la tendencia que presenta la demanda de la estructura $(\hat{D})$ con la intensidad $(S a / g)$. En estas figuras se puede observar que mientras más grande es el intervalo de tiempo (por consecuencia más daño estructural acumulado), los valores de la mediana de la demanda se incrementan. La expresión que ajusta a la mediana de la demanda $\hat{D}$, es la misma para los tres instantes de tiempo en estudio. 


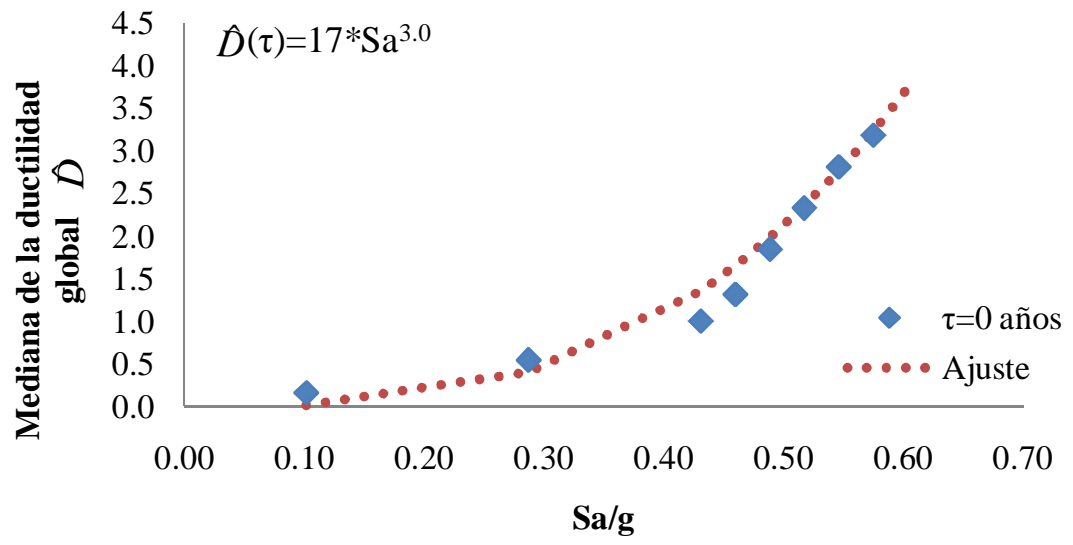

(a) Estructura sin daño acumulado

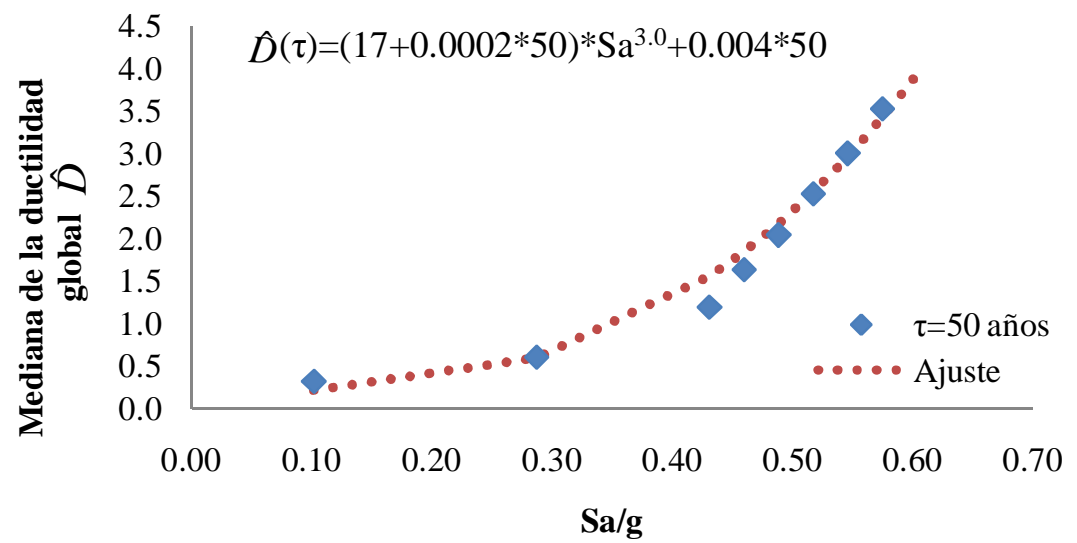

(b) 50 años

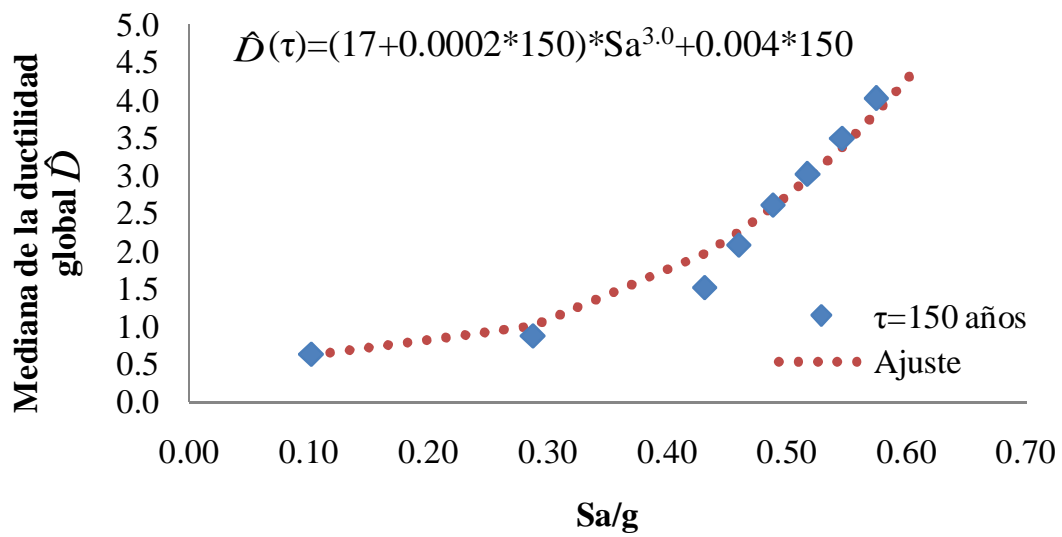

(c) 150 años

Figura 10. Ajuste de las funciones que representan la mediana de la demanda $\hat{D}$, al término de los intervalos de tiempo de 0,50 y 150 años. 
En la tabla 4 se presentan las desviaciones estándar del logaritmo natural . Cabe mencionar que los valores de las desviaciones estándar del logaritmo natural de la demanda se incrementan a medida que el intervalo de tiempo crece. Esto se debe a que mientras más grande es el intervalo de tiempo, mayor es la probabilidad de que la estructura esté sometida a más movimientos sísmicos intensos, por lo que se acumula mayor daño estructural. Esto se refleja en que la estructura presenta un número mayor de elementos con articulaciones plásticas y/o estas desarrollan mayor ductilidad ante la acción de dichos movimientos. El comportamiento dinámico no lineal de dichos elementos correspondientes a estructuras sujetas a un conjunto de historias aleatorias de aceleración da lugar a una mayor variabilidad de la respuesta estructural.

Tabla 4. Desviaciones estándar del logaritmo natural de la demanda estructural, $\sigma_{l n D}$.

\begin{tabular}{cc}
\hline $\begin{array}{c}\text { Tiempo } \\
\text { (años) }\end{array}$ & $\sigma_{l n D}$ \\
\hline 0 & 0.12 \\
50 & 0.15 \\
150 & 0.25 \\
\hline
\end{tabular}

\section{Obtención de parámetro $r$}

El parámetro $r$ corresponde a la curva de peligro sísmico de la forma , donde $k$ y $r$ son constantes que se ajustan a la forma de la CPS para la intensidad de interés. En la figura 11 se muestran los ajustes a la $C P S$ correspondiente a $\mathrm{T}=1 \mathrm{~s}$ del sitio SCT. En este estudio se analizan resultados correspondientes a dos estados límite: a) estado límite de servicio para el que $\mu=1$, y b) estado límite cercano al colapso, para el que $\mu=2.8$. Los valores de las ductilidades para cada estado límite se estimaron a partir de la curva de capacidad sin daño (figuras $5 a$ y 5 b).

Con el valor de estos estados límite seleccionados $(\mu=1$ y $\mu=2.8)$, se ingresa a las curvas de ajuste de la mediana de la demanda $\hat{D}$ para los tres instantes de tiempo 0,50 y 50 años después de construida la estructura versus (figuras $10 \mathrm{a}, 10 \mathrm{~b}$ y 10c) y se obtiene el valor de la intensidad asociada a dichos valores de demanda. A partir de la intensidad obtenida, se ingresa a la curva de peligro sísmico (Alamilla, 2001) correspondiente al periodo fundamental de la estructura $(\mathrm{T}=1 \mathrm{~s})$ (ver figura 11).

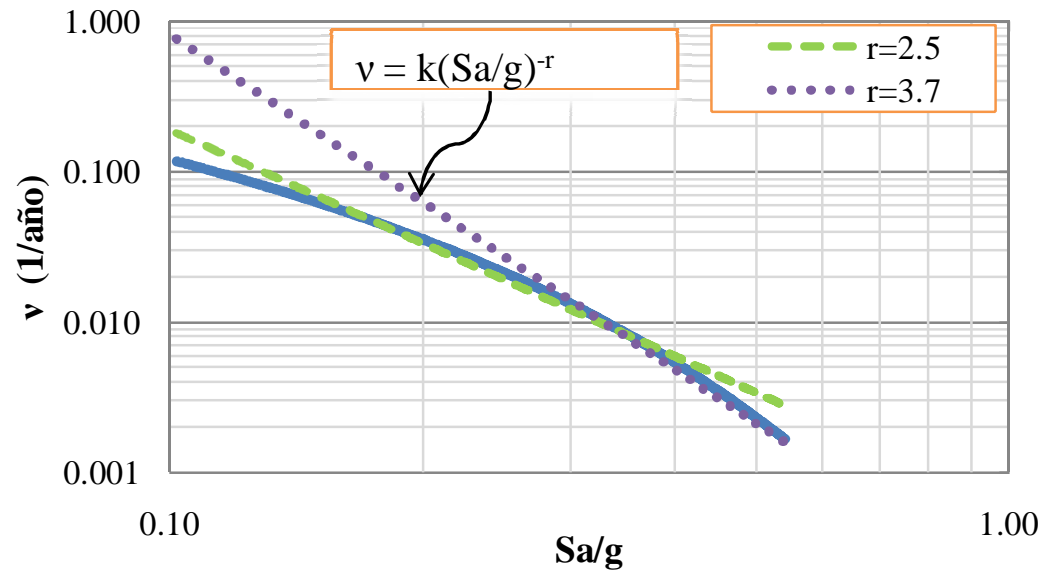

Figura 11. Ajuste de los valores $r$ en la curva de peligro sísmico sitio SCT para una sistema con periodo de vibración $\mathrm{T}=1 \mathrm{~s}$. 


\section{Obtención del número de fallas en un intervalo de tiempo}

En lo que sigue se compara el número de fallas de la estructura en el tiempo $\left(\eta_{F, T}\right)$ considerando solamente la variación en el tiempo de la capacidad estructural (Torres, 2006; Torres y Ruiz, 2007) (ec. 12) versus el número de fallas en el tiempo $\left(\eta_{F, T}\right)$ considerando, la variación en el tiempo de la capacidad y de la demanda dada una intensidad (ec. 22). Se supone que las incertidumbres epistémicas asociadas con la demanda $\sigma_{U D \mid \tau}^{2}$, y con la capacidad $\sigma_{U C \mid \tau}^{2}$, son iguales a 0.2 .

Los valores de $\alpha$ y $\beta$, que están relacionados con la tendencia que tiene la mediana de la capacidad $\hat{C}$ con la intensidad, se obtienen a partir de la figura 7. Los valores $b, e, f$ y g que están relacionados con el incremento de la mediana de la demanda $\hat{D}$ con la intensidad, se obtienen de las figuras $10 \mathrm{a}, \mathrm{b}$ y c para 0,50 y 150 años, respectivamente.

A partir de los valores de $\alpha, \beta, e, f, g, r$ y $b$ que se obtienen para cada estado límite, se calcula la función hipergeométrica (que está implícita en la ec. 23) y se obtiene una expresión de factor de corrección $\Omega_{0}(t, \Delta t)$ para cada estado límite, como sigue:

- Estado límite de servicio:

$$
\begin{aligned}
& \Omega_{0}(t, \Delta t)= \\
& -\frac{b\left(1.0005+1.63797 * 10^{-6} t\right)\left(\frac{(e+f t) \beta}{-f \alpha+e \beta}\right)^{-\frac{r}{b}}(\alpha+t \beta)}{(b-r) \beta}+ \\
& \frac{b\left(\frac{\alpha+t \beta}{e+f t}\right)^{\frac{r}{b}}\left(1.0005+1.63797 * 10^{-6}(t+\Delta \mathrm{t})\right)\left(\frac{\beta(e+f(t+\Delta \mathrm{t}))}{-f \alpha+e \beta}\right)^{-\frac{r}{b}}(\alpha+\beta(t+\Delta \mathrm{t}))\left(\frac{\alpha+\beta(t+\Delta t)}{e+f(t+\Delta t)}\right)^{-\frac{r}{b}}}{(b-r) \beta}
\end{aligned}
$$

- Estado límite cercano al colapso:

$$
\begin{aligned}
& \Omega_{0}(t, \Delta t)= \\
& -\frac{b\left(0.99891-3.59343 * 10^{-6} t\right)\left(\frac{(e+f t) \beta}{-f \alpha+e \beta}\right)^{-\frac{r}{b}}(\alpha+t \beta)}{(b-r) \beta}+ \\
& \frac{b\left(\frac{\alpha+t \beta}{e+f t}\right)^{\frac{r}{b}}\left(0.99891-3.59343 * 10^{-6}(t+\Delta \mathrm{t})\right)\left(\frac{\beta(e+f(t+\Delta \mathrm{t}))}{-f \alpha+e \beta}\right)^{-\frac{r}{b}}(\alpha+\beta(t+\Delta \mathrm{t}))\left(\frac{\alpha+\beta(t+\Delta \mathrm{t})}{e+f(t+\Delta \mathrm{t})}\right)^{-\frac{r}{b}}}{(b-r) \beta}
\end{aligned}
$$

En las figuras 12a y $12 \mathrm{~b}$ se presentan las relaciones entre los factores de corrección $\frac{\Omega_{0}(t, \Delta t)}{\Omega(t, \Delta t)}$ obtenidos con la ecs. 23 y 14. Dichas relaciones corresponden a los estados límite de servicio y cercano al colapso, respectivamente.

En las tablas 5 y 6 se muestra el número de fallas esperado al término de un intervalo de tiempo $\left(\bar{\eta}_{F, T}\right)$ para los estados límite de servicio y cercano al colapso, respectivamente. Para ello se consideraron la ec. 12 (Torres, 2006; Torres y Ruiz 2007) y la ec. 22 (este estudio). 


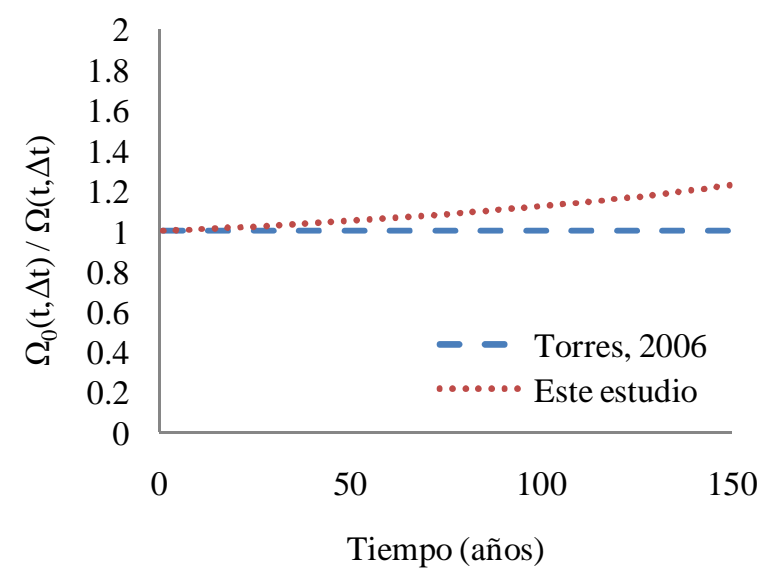

a) Estado límite de servicio

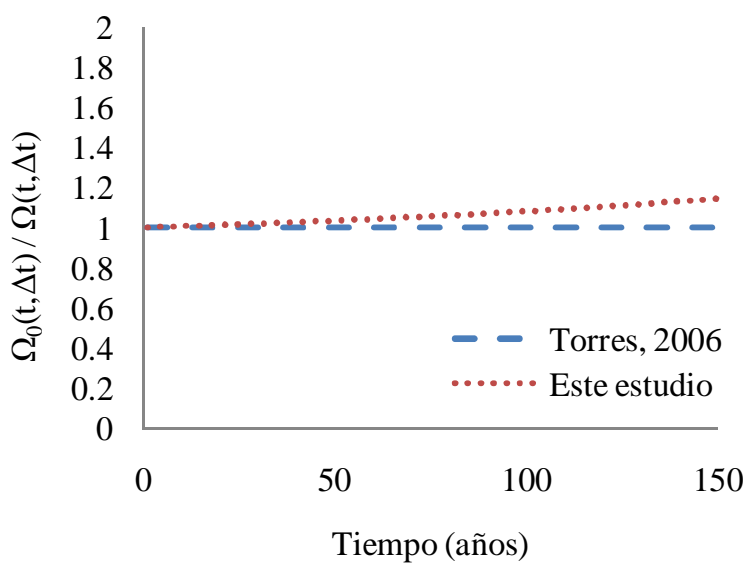

b) Estado límite cercano al colapso

Figura 12. Relación de factores de corrección

Tabla 5. Valores esperados del número de fallas al término de diferentes intervalos de tiempo para el estado límite de servicio.

\begin{tabular}{ccc}
\hline \multicolumn{3}{c}{ Estado Límite de Servicio } \\
\hline $\begin{array}{c}\text { Intervalo } \\
\text { (años) }\end{array}$ & (ec. 12) & (ec. 22) \\
\hline 0 & 0.0005 & 0.0005 \\
50 & 0.1460 & 0.1511 \\
150 & 0.4944 & 0.5660 \\
\hline
\end{tabular}


Tabla 6. Valores esperados del número de fallas al término de diferentes intervalos de tiempo para el estado límite cercano al colapso.

\begin{tabular}{ccc}
\hline \multicolumn{3}{c}{ Estado Límite Cercano al Colapso } \\
\hline $\begin{array}{c}\text { Intervalo } \\
\text { (años) }\end{array}$ & $\begin{array}{c}\bar{\eta}_{F, T} \\
\text { (ec. 12) }\end{array}$ & $\begin{array}{c}\bar{\eta}_{F, T} \\
(\mathrm{ec} .22)\end{array}$ \\
\hline 0 & 0.0003 & 0.0003 \\
50 & 0.0934 & 0.0981 \\
150 & 0.3430 & 0.4211 \\
\hline
\end{tabular}

Las tablas 5 y 6 muestran que el considerar la variación de la demanda estructural (dada la intensidad) con el tiempo, en el cálculo del número esperado del número de fallas al final de cierto intervalo de tiempo, $\bar{\eta}_{F, T}$, cobra mayor importancia a medida que el intervalo de tiempo crece. Esto ocurre tanto para el estado límite de servicio como para el estado límite cercano al colapso.

\section{CONCLUSIONES}

Se propuso un criterio para evaluar la confiabilidad estructural considerando la variación en el tiempo de la capacidad y de la demanda para una intensidad dada por medio de expresiones matemáticas cerradas. La confiabilidad se expresa en términos del número esperado de fallas en un intervalo de tiempo.

Los resultados obtenidos en el ejemplo que se presenta hacen ver la importancia que tiene considerar el daño acumulado en las estructuras en la evaluación de la confiabilidad sísmica estructural.

El ejemplo de aplicación hace ver que el deterioro estructural de las propiedades mecánicas tiene influencia no solamente en la capacidad estructural sino también en la demanda (como función de la intensidad). Esto se reflejó en el hecho que el número esperado de fallas en un intervalo de tiempo, $\bar{\eta}_{F, T}$, resultó mayor cuando se incluyó en el análisis el efecto de la variación de la demanda estructural en el tiempo que cuando solamente se consideró deterioro de las propiedades mecánicas en la capacidad estructural. La diferencia entre ambos casos resultó mayor a medida que el intervalo de tiempo en consideración era más grande.

El valor de $\bar{\eta}_{F, T}$ se incrementa un $3.5 \%$ y un 5\%, para los estados límite de servicio y de colapso, respectivamente, cuando el intervalo considerado es de 50 años; sin embargo, dichos incrementos son de $14.5 \%$ y $22.7 \%$, para los estados límite de servicio y cercano al colapso, respectivamente, cuando el intervalo es de 150 años. Estos resultados muestran que cuando no se considera la variación de la demanda estructural con el tiempo se subestima la tasa de excedencia de la estructura para cierto intervalo de tiempo, y por lo tanto se sobre-estima su confiabilidad. Esto tiene repercusiones indeseables, por ejemplo para el caso en que se tomaran decisiones sobre el mantenimiento estructural basadas en riesgo, o si se realizara un diseño estructural basado en confiabilidad o en optimación de costos esperados en el ciclo de vida de la estructura.

El criterio propuesto es aplicable a cualquier tipo de estructura (p.ej., plataformas marinas, torres de transmisión, etc), y puede adaptarse para considerar solicitaciones asociadas a diferentes tipos de fenómenos naturales (por ejemplo oleaje, viento, etc). 


\section{AGRADECIMIENTOS}

Los autores desean agradecer a un revisor anónimo sus valiosos comentarios, que enriquecieron este escrito. También agradecen a CONACYT, y a la DGAPA de la UNAM dentro del Programa de Apoyo a Proyectos de Investigación e Innovación Tecnológica (PAPIIT) su apoyo para la elaboración de esta investigación. El diseño del edificio que se presenta en el ejemplo fue realizado por García Jarque Ingenieros, S.C.

\section{REFERENCIAS}

Alamilla, J L (2001), "Criterios de diseño sísmico basados en confiabilidad para estructuras aporticadas", Tesis para obtener el grado de Doctor en Ingeniería, DEPFI, UNAM.

Braverman, J I, C A Miller, C H Hofmayer, B R Ellingwood, D J Naus, y T Y Chang (2004), "Degradation assessment of structures and passive components at nuclear power plants", Nuclear Engineering and Design, marzo 2004, Vol. 228, No 1-3, pp. 283-304.

Campos, D y L Esteva (1997), "Modelo de comportamiento histerético y de daño para vigas de concreto reforzado", XI Congreso Nacional de Ingeniería Sísmica, Veracruz, México.

Chan, S M (2003), "Influencia del escalamiento de movimientos sísmicos de banda estrecha en la evaluación de la confiabilidad de edificios", Tesis de Maestría, Programa de Maestría y Doctorado en Ingeniería, UNAM.

Chan, S M, S E Ruiz y M A Montiel (2005), "Escalamiento de acelerogramas y número mínimo de registros requeridos para el análisis de estructuras", Revista de Ingeniería Sísmica, Vol. 72, pp 1-24.

Cornell, C A (1968), "Engineering seismic hazard analysis", Bulletin of the Seismological Society of America, Vol. 58, No.5, pp. 1583-1606.

Cornell, C A (1996), "Calculating building seismic performance reliability: A basis for multi-level design norms", $11^{\text {th }}$ World Conference on Earthquake Engineering, Paper 2122.

Cornell, C A, F Jalayer, R O Hamburger, y D A Foutch (2002), "The probabilistic basis for the 2000 SAC/FEMA steel moment frame guidelines", ASCE Journal of Structural Engineering; Vol. 128, No.4, pp. 526-533.

Cruz, C (2007), "Análisis de confiabilidad de edificios de concreto en zonas sísmicas considerando la acumulación de daño estructural durante la vida de servicio", Tesis para obtener el grado de Maestro en Ingeniería, Programa de Maestría y Doctorado en Ingeniería, UNAM.

Díaz, O J y L Esteva L (1997), "Confiabilidad de estructuras y daño acumulado", Memorias Séptimas Jornadas Chilenas de Sismología e Ingeniería Antisísmica y Primer Congreso Iberoamericano de Ingeniería Sísmica, La Serena, Chile, pp 589-598.

Esteva, L (1968), "Bases para la formulación de decisiones de diseño sísmico", Tesis de Doctorado, Facultad de Ingeniería, UNAM, México.

FEMA 351 (2000), "Recommended seismic evaluation and upgrade criteria for existing welded steel moment-frame buildings", Report No. FEMA-351, SAC Joint Venture, Washington, D.C.

Galambos, T V, B R Ellingwood, J G McGregor, y C A Cornell (1982), "Probability based load criteria: assessment of current design practice", ASCE Journal of Structural Division, Vol. 108, No. ST5, pp. 959-977.

García Jarque Ingenieros S. C., (2004), Comunicación personal, México D.F. 
Hong, H P y E Rosenblueth (1988), “The Mexico earthquake of september 19, 1985- model for generation of subduction earthquakes", Earthquake Spectra, Vol. 4, No. 3, pp. 481-497.

Gusella, V (1998), "Safety estimation method for structures with cumulative damage", Journal of Engineering Mechanics, Vol. 124, No. 11, pp. 1200-1209.

Hamamoto, T (1996), "Stochastic long-term damage prediction of multi-story buildings", Eleventh World Conference on Earthquake Engineering, Acapulco, México.

Jalayer, F y C A Cornell (2003), "A technical framework for probability-based demand and capacity factor (DCFD) seismic formats", Technical Report PEER 2003/08, Pacific Earthquake Engineering Center, University of California, Berkeley.

Ma, S M, V V Bertero y E P Popov (1976), "Experimental and analytical studies on the hysteretic behavior of reinforce concrete rectangular and t-beams", Earthquake Engineering Research Center, Reporte No. EERC 76-2, Universidad de California, Berkeley.

Montes-Iturizaga, R, E Heredia-Zavoni, y L Esteva (2003), "Optimal maintenance strategies for structures in seismic zones", Earthquake Engineering and Structural Dynamics, Vol. 32, pp. 245-264.

Montiel, M A (2005), "Confiabilidad implícita en estructuras convencionales como base para establecer criterios para el diseño sísmico de estructuras reforzadas con disipadores de energía", Tesis para obtener el grado de Doctor en Ingeniería, Programa de Maestría y Doctorado en Ingeniería UNAM.

Montiel, M A, S E Ruiz y M A Torres (2003), "Comparison of two alternative methods for estimating annual mean seismic failure rates of building frames", Coloquio Internacional: Risque, Vulnérabilité \& Fiabilité Dans La Construction, Vers une Reduction des Désastres, 11 y 12 de octubre, Alger-Algérie, pp 524-534.

RCDF (2004), Normas Técnicas Complementarias para Diseño por Sismo, Diario Oficial de la Federación

Rivera, J L (2001), "Respuesta sísmica probabilística de sistemas estructurales simples no lineales", Tesis de Maestría en Ciencias, Sección de Estudios de Posgrado, ESIA, IPN.

Scribner, C F y J K Wight (1978), "Delaying shear strength decay in reinforced concrete flexural members and large load reversals", Reporte No. UMEE 78R2, Departament of Civil Engineering, University of Michigan, Michigan.

Rosenblueth, E y L Esteva (1972), "Reliability basis for some Mexican codes", ACI Publication SP-31, pp. 1-41.

Shome, N y C A Cornell (1999), "Probabilistic seismic demand analysis of nonlinear structures", Report No. RMS-35, Department of Civil and Environmental Engineering, Stanford University.

Tolentino, D (2009), "Confiabilidad de edificios considerando la influencia del daño acumulado causado por sismos", Tesis para obtener el grado de Maestro en Ingeniería, Programa de Maestría y Doctorado en Ingeniería UNAM.

Torres, M A (2006), "Criterios costo/beneficio para la inspección y mantenimiento de plataformas marinas", Tesis para obtener el grado de Doctor en Ingeniería, Programa de Maestría y Doctorado en Ingeniería UNAM.

Torres, M A, S E Ruiz y U Elena (2006), "Evolución en el tiempo de la confiabilidad sísmica debida al deterioro estructural", XV Congreso Nacional de Ingeniería Estructural, Puerto Vallarta, México.

Torres, M A y S E Ruiz (2007), "Structural reliability evaluation considering capacity degradation over time”, Engineering Structures, Elsevier, Vol. 29, pp. 2183-2192. 
Townsend, W H y R D Hanson (1977), "Reinforced concrete connection hyseresis loops", Publicación ACI SP53-13, pp. 351-370.

Uzumeri, S M (1977), "Strength and ductility of cast-in-place beam-column joints", Publicación ACI SP53-13, pp. 283-350.

Vamvatsikos, D y C A Cornell (2002), "The incremental dynamic analysis and its application to performance-based earthquake engineering", Proceedings of the $12^{\text {th }}$ European Conference on Earthquake Engineering, Paper 479, London, UK.

Vásquez, A (2010), "Funciones de daño acumulado para edificios de concreto reforzado", Tesis para obtener el grado de Maestro en Ingeniería, Programa de Maestría y Doctorado en Ingeniería UNAM.

Wang, M L y S P Shah (1987), "Reinforced concrete hysteresis model based on the damage concept", Earthquake Engineering and Structural Dynamics, Vol. 15, pp. 993-1003.

Wen, Y K (1995), “Building reliability and code calibration”, Earthquake Spectra, Vol. 11, pp 269-296. 